\title{
The molecular economics of the long neuron
}

\author{
IAIN H. M. SMART \\ Queen's College, University of St. Andrews, Dundee, Scotland
}

KURZFASSUNG: Die molekulare OKonomie der langen Neuronen. Im Gegensatz zu anderen Zellen ist ein Neuron in der Lage, durch Veränderung der Kern-Plasma-Relation progressiv zu wachsen. Dieses Phänomen wurde untersucht, wobei der Eiweißstoffwechsel einer Zelle als limitierender Faktor für die Erhaltung der normalen Kern-Plasma-Relation angesehen wurde. Es wurde ein Modell eines Systems konstruiert, mit voneinander abhängigen Molekülen verschiedener Halblebenszeiten, welche unter den Bedingungen molekularen Austausches operieren, die den Proteinstoff wechsel $\mathrm{zu}$ beherrschen und einzuschränken scheinen. Dann wurde geprüt, auf welche Weise dieses System sich durch Anstieg der Molekulzahlen auszudebnen vermag. In einer weitgehend dem Muster des unbeschränkten Wachstums der langen Neuronen ähnelnden Situation wurde dem Mangel an Molekülen mit kurzer Halblebenszeit (diese wurden in sich ausdehnenden Systemen gefunden) durch Überführung ähnlicher Moleküle aus benachbarten, nicht ausgedehnten Systemen begegnet. Das ausgedehnte System neigte aber dazu, "Molekülschulden" auf sich zu laden, deren Höhe abhing von dem Ausmaß der Systemausdehnung. Diese Sadvverhalte stimmten überein mit vielen beobachteten histologischen, biochemischen und physiologischen Charakteristika der Nervengewebe. Es wurde daher gefolgert, daß Modelle dieser Art nützlich sein können hinsichtlich einer Korrelation morphologischer und biochemischer Befunde und der Planung geeigneter Experimente.

\section{INTRODUCTION}

The long neuron is the unique example of a mononucleate diploid cell which is capable of growing indefinitely by progressively changing its nucleo-cytoplasmic ratio (Fig. 1C). In all other cell types, whether protozoan, metazoan or plant, progressive accumulation of metabolically active cytoplasm is accompanied by an increase in the number of nuclei (Fig. 1A), or an increase in the degree of ploidy of a single nucleus (Fig. 1B) in such a way that the nucleo-cytoplasmic ratio is maintained within the original order of magnitude. It is difficult to explain the unique position of the long neuron in terms of classical cell biology, nor does it seem a problem which is amenable to experimental solution, unless, as a preliminary, some theoretical groundwork is established. This communication is an attempt to rearrange certain established data in such a way that a pattern emerges which can account for the growth of a neuron in terms of the progressive modification of the metabolism of a columnar neural plate cell. The problem is approached by shifting the emphasis from the morphological conception of a cell as a quantity of nucleated cytoplasm to one which recognizes a cell as a metabolic entity subject to certain limitations governing the turnover of its component molecules. It is proposed to treat cell grow th as a problem in molecular econo- 


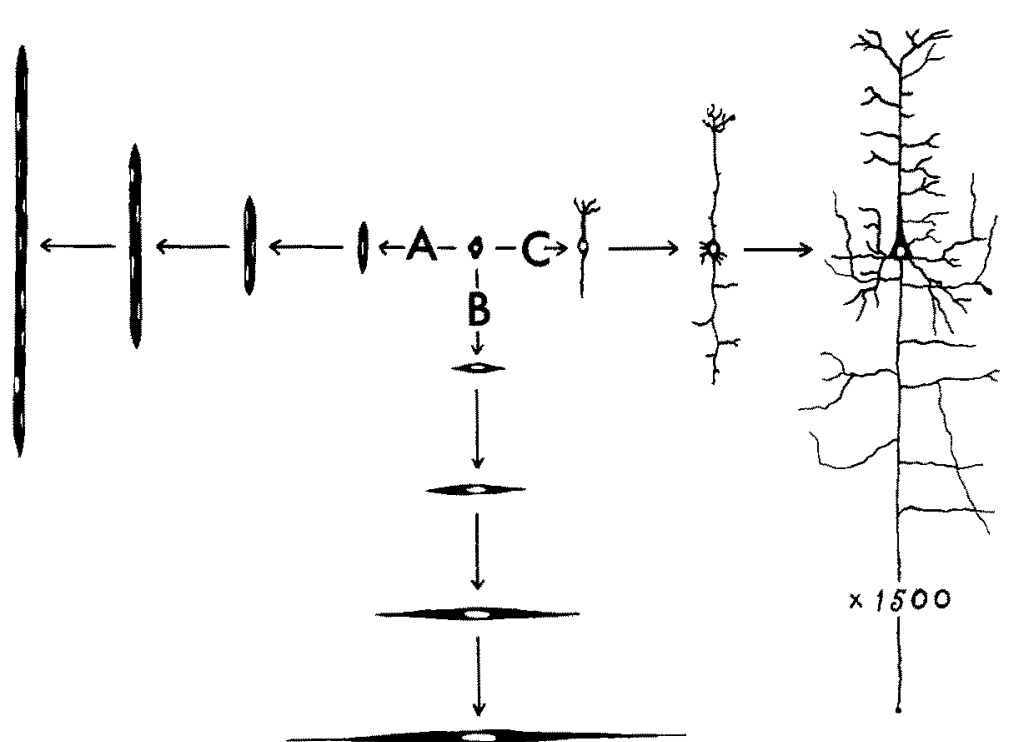

Fig. 1: Diagrammatic representation of three basic methods of cell growth. The mononucleate diploid cell at the centre can grow either by increasing the number of its nuclei as in the striated muscle cells indicated by arrow $A$, or by increasing the size and degree of ploidy of a single nucleus as seen in the growth of smooth muscle cells of the gravid uterus depicted by arrow $B$. Nerve cells grow by progressively changing their nucleo-cytoplasmic ratio, as indicated by arrow $C$ which shows a developmental sequence adapted from Cajal. A humancortical pyramidal cell if represented in full would extend 1500 times the distance shown here

mics and rephrase the question as follows: how does the metabolic system represented by the neural plate cell at $C$ in Figure 1, which has a cytoplasmic mass (number of molecules) similar to that of the other somatic cells of the organism, accumulate and maintain further molecules until it resembles the cortical pyramidal cell at the extreme right with a surface area and cytoplasmic mass many hundreds of times their original value?

\section{RESULTS AND DISCUSSION}

The molecules which make up a cell are usually classified according to their chemical composition, e. g. protein, fat, carbohydrate, or according to their function, e. g. different classes of enzymes, hormones, participants in different metabolic cycles and so on. A third classification of molecules is possible and that is according to their stability or "half life" within the cell, since it is useful to know not only the structure and function of a particular molecule, but also how long it remains in the cell before it is catabolised or eliminated. A classification of this nature is of particular interest when applied to the complex molecules which a cell synthesizes from structurally simpler molecules derived from its environment. For present purposes the different species or complex molecules comprising a cell are grouped into three classes defined according to their stabilities as follows:- 
Class 1 Molecular species which, once synthesized, are stable, or whose turnover is relatively slow, and whose stability is little affected by variations in the metabolic activity of the cell as a whole.

$\mathrm{Cl}$ ass 2 Molecular species which are intermittently labile, that is, under certain metabolic conditions they are stable according to the definition of Class 1 , but under other metabolic conditions they are subject to turnover.

Class 3 Molecular species which have a relatively short "half-life" under all metabolic conditions and are constantly being resynthesized by the cell. Class 3 molecules subject to periodic increases in their rate of turnover would share some of the properties of Class 2.

This classification can be usefully applied to the protein component of a cell's population of complex molecules. Protein biochemists acknowledge a wide range of half-lives among different protein species (TARver 1954), indicating a spectrum of stabilities between Class 1 and Class 3 . The existence of Class 2 proteins is suggested by the fluctuation of intracellular enzyme levels during different phases of metabolic cycles (TARver 1954).

The mechanism of protein synthesis, moreover, seems to impose certain limitations on the accumulation of molecules of the type defined under Class 3 , and presumably also secondarily on the members of the other two classes. According to the recent work of molecular biologists based on the Watson-Crick model and theory (WATSON \& CRICK 1953a, b) the synthesis of each protein species involves the serial participation of one DNA template and a set of RNA templates, the specificity of the process depending on the sequence in which the component DNA and RNA bases are arranged. This introduces certain limitations. Multistep chemical reactions such as those involved in template synthesis ultimately reach a certain maximum velocity. Thus a particular template synthesizing unstable products is able to form and maintain a population of molecules whose maximum number is limited by their rate of synthesis and half-life (e. g. for a fixed rate of synthesis, the longer the half-life of the synthesized molecules, the more numerous the resultant population). DNA templates are considered to be stable in that, as a general rule, they are not subject to metabolic turnover (see review by VENDRELY 1955), and also in the sense that they do not readily change their specificity by rearranging the order of their base sequence. A further peculiarity is that, in general, a cell is unable to synthesize single DNA templates as required. A particular template can only be doubled at the expense of replicating the cells entire DNA template population. This is indicated by the experimental work of Boivin, Vendrely \& Vendrely (1948) and Mirsky \& Ris (1949) who found that the average amount of DNA per chromosome set is constant for all the diploid cells of an organism and is fixed and constant for each particular species of animal. Variations of nuclear DNA content when they do occur tend to be in multiples of the diploid amount, (see also reviews by VENDRELY \& VENDRELY 1956 and by Mrrsky \& Osawa 1961). DNA synthesis, therefore, tends to be intermittant and to involve the replication of the entire population of DNA molecules in the cell. It is an event which may or may not be followed by the appearance of a mitotic figure and cell division. The synthesis of RNA and protein, on the other hand, is a continuous process, which indicates that both have components which are subject to 
continuous turnover. Each DNA template could, therefore, be considered to be related to a certain maximum population of RNA and protein molecules; a DNA template associated with an RNA and protein species with short half-lives would thus have a smaller population of dependant molecules than one concerned with the synthesis of stable products. The metabolism of a diploid cell may, therefore, reach equilibrium when the maximum attainable population of an essential short-lived protein species becomes insufficient to meet the increasing metabolic demands of the growing cell.


Fig. 2: $A$ represents the basic input-output model described in the text. $B, C$ and $D, E, F$ represent the variation which permit its indefinite growth

Let us consider the properties of a molecular economy of this type by means of a simplified model. Figure $2 \mathrm{~A}$ is intended to represent a molecular system which is assumed to have the following three primary properties: first, the system contains different species of molecules, at least one of which is subject to continuous turnover; second, the different species of molecule cannot remain in the system unless a certain ratio exists between them; third, it is possible to add to each molecular species from sources which can deliver at a certain maximum rate. The system in Figure $2 \mathrm{~A}$ is pictured as having three different species of molecules with one source of new molecules for each species. The sources are represented by numbered arrows which, in this case, are located within the community whose limits are defined by a circumscribing line. For the sake of simple example, the numerical values arbitrarily chosen for the primary limitations are, that the different molecular species cannot remain in the system for more than one second unless a one-to-one ratio exists between them, and that each source can add to the system at a maximum rate of one molecule per second. The first species of molecule, represented by solid black roundels, is stable and 
corresponds to the species already defined under Class 1 and is able to accumulate in the system provided the one-to-one ratio with the other molecular species is maintained. The second species, represented by the black and white semicircles, is intermittently labile as defined previously under Class 2: under one set of conditions it is stable and under a second set it is subject to the loss of two molecules in ten each second of time. The third species, represented by open circles, is unstable and subject to a continuous loss of one molecule in ten each second. In the diagram the molecules are arranged in rows for simplicity of illustration, but can also be pictured as being randomly distributed in the system. Let us say that the system has reached equilibrium when there are ten molecules in each species. A certain minimal amount of oscillation in numbers can be expected in view of the one second interval in which a molecule can remain in the system without a one to one ratio existing between each molecular species and the possibility of asynchronous addition of molecules to each species. Oscillation will be more pronounced when the Class 2 molecules change from the stable to the unstable state. For example, if the system runs for two seconds under Class 2's second set of conditions, at the end of this time four molecules of Class 2 will have left the system and two will have entered. The population will now have dropped to 8 . At the end of the third second another Class 2 molecule will have been supplied, but one molecule from Class 1 will have been obliged to leave the system because the one to one relationship has not been maintained for over a second. The system will then contract and reconstitute itself during the fourth second.

The model can now be used as a primary oversimplification to indicate the general pattern of the limitations under which a system of this type operates, and the possible ways in which they may be overcome. The property presently being investigated is that of growth by increase in the number of molecules in the system. The primary properties under which the system operates exclude the possibility that indefinite growth can occur by (1) progressively increasing the rate of addition from a single source or by (2) changing the numerical relation between the species, i. e. the ratio of Class 1 and 2 to Class 3 molecules changing progressively from $1: 1: 1$ to $2: 2: 1,3: 3: 1$ and so on, or by (3) rendering stable or by eliminating the labile species of molecule. The first limitation rests on the fact that a multistep chemical reaction, such as a template-mediated synthetic reaction, ultimately reaches a maximum velocity. The second rests on the fact that if the labile molecules are an energy source the energy they contain is finite, or if their function is catalytic, a certain minimum ratio of catalyst to reactants must be maintained. The third rests on the fact that in all cells investigated, including the long neuron, continuous protein turnover has been demonstrated even when cell growth is absent (LEBLond et al. 1957).

Such a system can thus grow indefin itely in only one way, namely increasing the number of sources of Class 3 molecules. Limited growth, however, may occur if the system has equilibrated below the possible upper limit set by the three primary limitations. 


\section{INDEFINITE GROWTH}

The number of sources may be increased by, (1) increasing the particular source required or by (2) increasing all three sources as a set or by (3) deriving the limiting molecular species from a source in a separate system.

Method (1) is depicted diagramatically in Figure $2 \mathrm{~B}$ which shows a system which has doubled its size by acquiring an additional single source of Class 3 molecules (indicated by extra Class 3 arrow). Indefinite repetition of this event would allow an indefinite increase in the number of molecules in the system. Growth, however, would be slow as the accumulation of Class 1 and 2 molecules would still be dependant on one set of sources each. In terms of the DNA template mechanism this can be compared with irregular increase in chromosome number (aneuploidy). Classical cell biology does not accept this as a normally occurring phenomenon. Nevertheless, it is possible that in situations where the demand on a particular part of the DNA template system is extreme, an individual DNA template may be duplicated without the duplication of the complete chromosome in which it is located. If this type of "microploidy" occurred the increase in DNA content would be small in relation to the total amount of DNA in the nucleus and, therefore, difficult to detect by chemical assay. Nevertheless, it should be technically possible to demonstrate if significant differences do occur between the DNA content of the nuclei of extremely large neurons and that of the animal's somatic cells. (The example offering the most extreme differences in size on earth would be the somatic cells and certain posterior root ganglion cells of a $30 \mathrm{~m}$ long adult blue whale.)

Method 2, in which the sources increase in complete sets, is represented by the model in Figure 2C, which shows a system which has equilibrated at double the original number of molecules by acquiring two sources for each molecular species. In growth by this method the number of molecules in an equilibrated system would be proportioned to the number of sets of sources. Growth would also be more rapid than in method 1 since the number of sources of Class 1 and 2 molecules would keep pace with those of Class 3 . An enlarged system would also be no more liable to oscillations in its molecular population than a small one.

In terms of the cell, this pattern of growth bears some resemblance to complete, repeated duplications of the chromosomes without cell division, which is observed to result in a rapidly growing, multinucleate (Fig. 1A) or polyploid cell (Fig. 1B), with a nucleocytoplasmic ratio remaining within the same order of magnitude as that of the cell of origin. Figure $2 \mathrm{C}$ thus provides a model which corresponds to the growth of such cells as muscle fibres, but not to that of the long neuron which is rarely other than mononucleate, and although a single nucleus may conceivably be tetraploid or even octaploid, it is certainly not centiploid or milliploid, as this method of growth would require.

Method 3 provides some interesting possibilities. Let us examine them with the help of Figure 2D which is intended to represent a molecular system similar to that in Figure $2 \mathrm{~A}$ except that it has doubled its total number of molecules by the progressive accumulation of Class 1 and Class 2 molecules. The one-to-one ratio has been maintained by deriving the necessary Class 3 molecules from two juxtaposed, but separate 
molecular systems designated $\mathrm{E}$ and $\mathrm{F}$ in Figure 2. Both systems $\mathrm{E}$ and $\mathrm{F}$ have five molecules of Class 3 within them and five they have each transferred to, and are maintaining in the extended system in Figure 2D. The one-in-ten loss per second can thus be made good and the system maintained in equilibrium. The system in Figure 2D is capable of progressive extension if sufficient supporting systems of the $E$ and $F$ type are present.

Now let us examine the properties of the Class 2 molecules in the extended system D. When stable they can contribute to the indefinite growth of the system. If subject to, say, a one second period when they lose two molecules in ten, the system will lose four molecules and gain one, and require a further three seconds to reconstitute itself. If the system $D$ doubles itself again to comprise a total of 40 molecules in each group, then during a one second period of turnover the Class 2 molecules will lose 8 of their population and require seven seconds for their reconstitution. This type of molecular debt will be encountered in any similar system which undergoes extension without a corresponding increase in its ability to replace molecules which are subject to intermittant loss, and is essentially a progressive exaggeration of the tendency to oscillate already described in the basic unextended system in Figure $2 \mathrm{~A}$. Growth of the "D" system would be slow as it would still be dependant on one set of sources for the synthesis of Class 1 and 2 molecules. If the Class 2 molecules entered a phase of turnover, growth would slow down or cease according to the numerical values under which the system was operating. The molecular debt contracted would be proportional to the number of Class 2 molecules at stake and the duration of turnover. In turn, the period of repayment would be proportioned to the size of the debt. Thus the larger the system, the larger the debt per unit time and the longer the period of repayment.

Consider next that a collection of extended systems of the type characterized by Figure 2D has been assembled, and that each system can enter a phase of turnover of Class 2 molecules, which can continue without the system contracting, until a certain fraction of the initial number of molecules has been lost, after which it is required to reaccumulate the lost molecules. When examined this collection is found to contain 100 small systems whose period of turnover-to-reaccumulation has a ratio of $3: 1$ and 100 large systems in which this ratio is $1: 3$. Let us suppose that the problem is to deploy the collection in such a way that the maximum number of systems is maintained in a state of turnover. The most efficient shift system which can be established will provide 75 simultaneously active small systems and 25 large. This indicates that what is true for the single system is also true for collections of systems, namely, the more numerous the larger systems in a collection, the larger the collective debt contracted per unit time, and the longer the total period of repayment.

The long neuron has at least a superficial resemblance to a single extended system of this nature and the nervous system as a whole to a collection of extended systems of varying size. For example, a neuron of more than a certain size is invariably invested over most of its non-synaptic surface by neuroglial "supporting" cells which are currently regarded as having their metabolisms very closely linked with that of the neuron. These cells could be equated with the subsidiary systems $\mathrm{E}$ and $\mathrm{F}$ in Figure 2. The work of HyDén (see review by HYDÉN 1960) has demonstrated that if a neuron is subject to chronic mild stimulation there is a decrease in certain enzymes in the 
perineuronal satellite cells and an increase in the same type of enzyme in the neuron body. In addition, a decrease in the RNA of the satellite cells is associated with an increase in neuronal RNA. In discussing this complex situation HYDÉN considers the possibility of molecular transfer taking place between satellite and neuron. One of the difficulties in this interpretation is that the increase in enzyme and RNA in the neuron is always less than the corresponding decrease in the satellite. However, a similar phenomenon would be encountered in the model in Figure $2 \mathrm{D}, \mathrm{E}$ and $\mathrm{F}$ if the transfer of molecules from systems $\mathrm{E}$ and $\mathrm{F}$ was occuring during a period of increased loss of Class 3 molecules in system $D$. The situation would be equivalent to adding water to an emptying bath tub - if one litre escaped per minute, the addition of 2 litres per minute would result in a net increase of only one litre per minute. In the case of RNA, however, HrDÉN found that, in addition to a quantitative disparity, the base proportions of the increased neuronal and decreased neuroglial RNAs did not match. This effect could also be simulated in a model in which two species of RNA of differing base composition were moving into the neuron at different rates and with different half-lives within the neuron.

There is a considerable body of literature to suggest that high molecular weight substances can pass through a cell membrane without being denatured in the process. Proteins are known to pass outwards through the membranes of the cells which have synthesized them as is seen in the case of the plasma proteins and the protein secretions of endocrine and exocrine glands. It is less generally accepted that large molecules such as proteins are able to pass inwards through a cell membrane. There is, however, a fairly extensive literature to suggest that this may occur. Workers with fluorescent antibodies interpret their findings on the basis that undenatured protein enters the cytoplasm and even nucleus of certain cells (Coons 1956) and embryologists attribute many of the induction phenomena to contact transmission of large molecules (protein and RNA) between cells (see reviews by Schechtman 1956 and Werss 1958). In this context it has been pointed out (SMART 1962) that in most stratified epithelia a specialized cell type is found which is concerned with mediating exchanges between the internal environment at the basement membrane and the more superficially situated cell strata, e. g. Sertoli cell in the seminiferous epithelium, follicular cell in the ovarian epithelium and melanocyte in the epidermis, to which could be added the glial cells of the neural epithelium. Thus some type of "transport" cell would seem to be usual rather than unusual in the organization of stratified epithelia. The suggestion that the neuroglia are able to solve the balance of payments problem of the neuron by transferring to it Class 3 proteins and'or protein sources (RNA), therefore, offends no general biological principle and can be entertained as a reasonable possibility.

A neuron process severed from its parent cell body invariably slowly degenerates; this indicates that the metabolic processes occurring in the nucleus and cell body are essential to the economy of the entire cell which presumably means that they contribute a molecular component to all cytoplasmic ramifications. That part, at least, of this component is protein in nature is suggested by the isotope studies of Droz \& LEBLOND (1962). This protein may have affinities with the Class 2 molecules, since on the one hand it would have to be stable in order to maintain its level of concentration 
in a cytoplasm of indefinite volume and yet, on the other, be unstable in order to require to be resynthesized.

It may be possible to resolve this paradox by comparing the neuron in its resting state with the extended molecular system in Figure 2D when its Class 2 molecules are stable, and when a nerve impulse passes with Figure 2D when its Class 2 molecules are subject to a 2 in 10 loss. This equates the passage of a nerve impulse with a metabolic event in which a pool of nucleus-dependant protein molecules is catabolized at a rate greater than they can be resynthesized. As the reaction is propagated throughout the cytoplasm the number of molecules broken down should bear a direct relation to the number of molecules in the pool, that is to the volume of the neuron. If the utilization of this molecular pool is even indirectly associated with the mechanism of impulse transmission then a neuron of more than a certain size subjected to the passage of impulses at more than a certain frequency will progressively deplete this pool of molecules until it is reduced to a level which is insufficient to support the passage of further impulses and the time required to reconstitute the pool to its original level would bear some direct relation to the size of the neuron.

A phenomenon of this sort could be regarded as an example of a "metabolic debt" occurring in an epithelial tissue comparable to the well known "oxygen debt" contracted by skeletal muscle when it functions at a metabolic rate faster than can be coped with by the circulating tissue fluids.

Let us next consider how a tendency to contract metabolic debts of this nature might affect the pattern of organization of a system of nerve cells. For example, a group of 100 small neurons whose turnover to reaccumulation ratio was, say, $3: 1$ would be able to muster 75 continuously active cells, whereas an equal number of long neurons in which this ratio was $1: 3$ would be able to muster only 25 continuously active cells. Thus a process requiring for its control the continuous participation of 75 neurons could be accommodated by a pool of 100 small neurons or 400 long neurons. Small neurons would, therefore, function most economically in the control of continuously occurring processes. Much of the continuous nervous activity of the body such as control of the internal economy (regulation of heart beat, respiration, temperature, etc.) is carried out by networks of short neurons typified by the brain stem reticular system. Multisynaptic systems of short neurons in addition to the reticular formation are numerous in the central nervous of man (CROSBY et al. 1962), and the retention of this "primitive" feature even in the most highly organized of terrestrial nervous systems may indicate that it is the most economical method of maintaining continuity of action.

Long neurons, on the other hand, eliminate synaptic delay and allow greater speed and specificity of nervous control over long distances. In more highly organized nervous systems these advantages may have offset any liability to accumulate metabolic debts, as this limitation can be compensated for, to some extent, by the formation of greater numbers of neurons. In homeothermic vertebrates, however, the number of neurons which an animal will possess during its life span is more or less established soon after birth or hatching, which imposes the following interesting limitation. Let us suppose that an animal has a hundred long neurons in its nervous system with a turnover to reaccumulation ratio of $1: 3$. These could be organized to function in different rotas 
between the following two extremes; either 25 out of 100 cells could function continuously, or 100 cells could function discontinuously in a $1: 3$ cycle, $i$. e. the animal could be $25 \%$ efficient $100 \%$ of the time or $100 \%$ efficient $25 \%$ of the time - the price of efficiency would appear to be discontinuous activity. A pattern of activity which alternated periods of activity with periods of rest would thus permit the most efficient deployment of an animals large nerve cells. An animal resting long motor cells would not move and an animal resting long sensory cells would not apprehend its environment. An animal in this state would bear some resemblance to a sleeping animal. Sleep may be a name for a state in which the passage of nerve impulses in a critical number of the larger neurons is reduced to a level which allows a particular group of molecules to be reaccumulated.

\section{LIMITED GROWTH}

Limited growth of the basic system in Figure 2A may occur if it has equilibrated below the possible upper limit set by the three primary limitations under which the system operates. It is difficult to assess how this factor has contributed to cell growth in general. However, it may be possible to discover particular situations in which a limiting factor becomes set at a higher level. For example the cause of the turnover

A
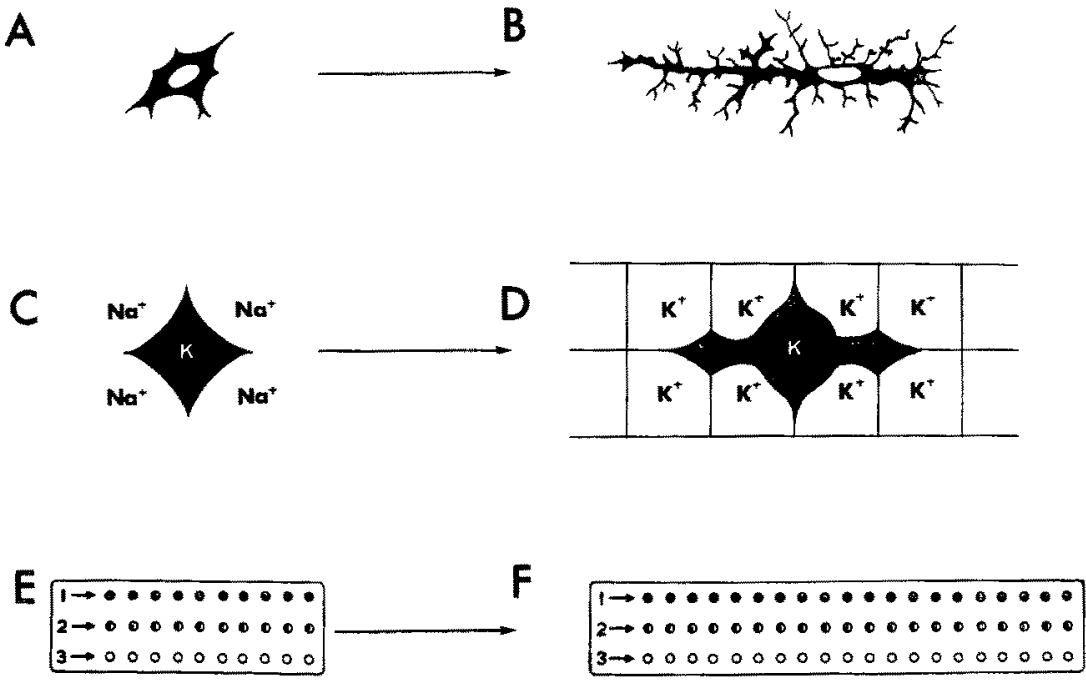

Fig. 3: At $A$ is a mesenchymal precursor cell of the microglia series. It has initially a nucleocytoplasmic ratio and surface area similar to the other cells of the organism. On entering the reduced space of the neural epithelium it increases its surface area and cytoplasmic mass and resembles the cell at $B$. A possible explanation of this phenomenon is given in $C$ and $D$. At $C$ is a diagrammatic representation of a mesendymal cell surrounded by extracellular fluid, the ionically different composition of which is indicated by the extracellular $\mathrm{Na}+$ contrasting with the intracellular $\mathrm{K}^{+}$. At $D$ the cell has moved into a reduced intercellular space epithelium and is surrounded by the ionically similar environment of its neighbouring cells represented here by the open squares containing the $K^{+}$symbol. Its metabolic expenditure on homeostasis is consequently reduced, permitting an increase in surface area. $E$ and $F$ represent this change in terms of the models described in the text 
of Class 3 molecules might be affected in such a way their half-life in the system was increased. In terms of the cell the basis of the lability of its Class 3 molecules may be that they form a pool similar to that suggested for the Class 2 molecules in Figure 2D which is utilized, however, in continuous processes rather than in intermittent events such as the passage of a nerve impulse. If one of these continuous processes is composed of the metabolic cycles concerned with the maintainance of the cell's homeostasis then this is a process whose rate would be reduced if the cell were in a "protected" environment.

Let us consider this possibility. The intercellular space of the central nervous system of both vertebrates and invertebrates is considerably smaller than that of the more loosely organized mesenchymal tissue. Electron microscope photographs indicate that the distance between the end of the electron-density of plasma membrane of one cell and the beginning of that of another is about $200 \AA$. A reduced intercellular space of this order of magnitude is characteristic of most surface epithelia and many of their derivatives. The physico-chemical forces existing in a space of this size are a subject of dispute (see reviews by WeIss 1958, Hydén $1960 \mathrm{~b}$ and DobBING 1961, 1963). There is evidence, however, which indicates that whatever material occupies this space, does not exert a homeostatic burden on the cell. (SMART 1962). The reduced space of stratified epithelium such as the central nervous system may, therefore, be a protected "ecological niche" wherein the metabolic cycles of a cell concerned with homeostasis are quantitatively reduced with a consequent decrease in the breakdown of participating molecules. This would permit the cell to grow until the next most active series of continuously occurring metabolic cycles was encountered. This change is depicted diagrammatically in Figure $3 \mathrm{~A}-\mathrm{F}$ which uses the microglial cell as an example. In terms of the model this is interpreted as a basic system (Fig. 3E) doubling its total population (Fig. 3F) by doubling the half-life of its Class 3 molecules. If a phenomenon of this nature exists, it would account for a contradiction in Figure 2 which indicates that the nucleo-cytoplasmic ratio of neuroglia cells diagrammatized by systems $\mathrm{E}$ and $\mathrm{F}$ in Figure 2 should be smaller than that of an average body cell, when in fact it is usually larger. Systems $E$ and $F$ in Figure 2 could thus be replaced by systems of the type in Figure 3F. This factor could, therefore, account for the initial extension of the long neuron and the complete extension of neuroglia and small neurons.

\section{SUMMARY}

1. Nerve cells grow by progressively changing their nucleo-cytoplasmic ratio, whereas growth in other cell types is associated with an increase in number of nuclei or degree of ploidy of a single nucleus in such a way that the nucleo-cytoplasmic ratio is maintained within its original order of magnitude.

2. Reasons are given for supposing that the primary factor in maintaining the nucleocytoplasmic ratio within its usual order of magnitude lies in the peculiar nature of protein metabolism which is keyed to a fixed number of DNA molecules.

3. An input-output model was constructed of a molecular system operating under similar limitations and its properties examined. 
4. By altering the values under which the system operated it was possible to examine the ways in which the system could grow. Patterns emerged for both limited and indefinite growth, both ultimately depending on the supply of molecules with a short half-life.

5. The pattern of indefinite growth which most nearly resembled that of the long neuron was one in which the deficiencies in labile molecules encountered by an extending system were met by transfer of similar molecules from adjacent unextended systems. The extended system, however, became liable to contract molecular debts whose magnitude was related to the degree of extension of the system.

6. A series of comparisons were drawn between the properties of the model and the observed morphological, biochenical and physiological characteristics of neural tissue. The coincidences were found to be sufficiently numerous to suggest that models of this type may be useful in correlating morphological and biochemical findings in the nervous system and in the design of experiments.

\section{LITERATURE CITED}

Borvin, A. R., Vendrely, R. \& Vendrely, C., 1948. L'acide désoxyribonucléique du noyal cellulaire, depositaire des caractères hẻréditaires; arguments d'ordre analytique. C. $R$. Acad. Sci., Paris 226, 1061-1063.

Coons, A. H., 1956. Histochemistry with labeled antibody. Int. Rev. Cytol. 5, 1-24.

Crosby, E. C., Humphrey, T. \& Lauer, E. W., 1962. Correlative Anatomy of the Nervous System. MacMillan, New York, $730 \mathrm{pp}$.

Droz, B. \& Leblond, C. P., 1962. Migration of proteins along the axons of the sciatic nerve. Science 137, 1047-1048.

DobBing, J., 1961. The blood-brain barrier. Pbysiol. Rev. 41, 130-188.

- 1963. The blood-brain barrier: Some recent developments. Guy's Hospital Reports 112, 267-286.

Hydén, H., 1960a. In: The Cell, Vol. 4, Chapter 5, Section 3 D. Academic Press, New York. $511 \mathrm{pp}$.

- 1960b. In: The Cell, Vol. 4, Chapter 5, Section 1 B. Academic Press, New York. 511 pp.

Leblond, C. P., Everett, N. B. \& Simmons, B., 1957. Sites of protein synthesis as shown by radioautography after administration of $\mathrm{S}^{35}$ - labeled methionine. Am. J. Anat. 101, 225-271.

Schechtman, A. M., 1956. Uptake and transfer of macromolecules by cells with special reference to growth and development. Int. Rev. Cytol. 5, 303-322.

SMArT, I., 1962. Neuroglia - an example of the evolution of transport cells in a tissue with reduced intercellular space. J. Anat. (Lond.) 96, 1.

- 1963. Evidence for the conditions existing in the $200 \AA$ intercellular space of epithelial tissues (Abstr.). J. Anat. (Lond.) 97, 302.

Tarver, H., 1954. In: The Proteins, Vol. 2 B, Chapter 26, Section 4. Academic Press, New York. 612 pp.

Vendrely, R., 1955. In: The Nucleic Acids, Vol. 2, Chapter 19, Section 3. Academic Press, New York. 580 pp.

- \& VendRely, C., 1956. The results of cytophotometry in the study of the deoxyribonucleic acid (DNA) content of the nucleus. Int. Rev. Cytol. 5, 171-198.

WATSON, J. D. \& CRICK, F. H. C., 1953a. A structure for deoxyribose nucleic acid. Nature, Lond. 171, 737-738.

- 1953b. Genetical implications of the structure of deoxyribonucleic acid. Nature, Lond. 171, 965-967.

WeIss, P., 1958. Cell contact. Int. Rev. Cytol. 7, 391-423. 